\title{
Children hear more about what is atypical than what is typical
}

\author{
Claire Bergey* \\ cbergey@uchicago.edu \\ Department of Psychology \\ University of Chicago
}

\author{
Benjamin C. Morris* \\ benmorris@uchicago.edu \\ Department of Psychology \\ University of Chicago
}

\author{
Daniel Yurovsky \\ yurovsky@cmu. edu \\ Department of Psychology \\ Carnegie Mellon University \\ University of Chicago
}

\begin{abstract}
How do children learn the typical features of objects in the world? For many objects, this information must come from the language they hear. However, language does not veridically reflect the world: People are more likely to talk about atypical features (e.g., "purple carrot") than typical features (e.g., "orange carrot"). Does the speech children hear from their parents also overrepresent atypical features? We examined the typicality of adjectives produced by parents in a large, longitudinal corpus of parent-child interaction. Across nearly 2000 unique adjective-noun pairs, we found parents' adjectives predominantly mark atypical features of objects, although parents of very young children are relatively more likely to comment on typical features as well. We then used vector space models to show that learning the typical features of common categories from linguistic input alone is challenging even with sophisticated statistical inference techniques.
\end{abstract}

Keywords: language input, language acquisition, childdirected speech, corpus analysis, word $2 \mathrm{vec}$

Children learn a tremendous amount about the structure of the world around them in just a few short years, from the rules that govern the movement of physical objects to the hierarchical structure of natural categories and even relational structures among social and cultural groups (Baillargeon, 1994; Legare \& Harris, 2016; Rogers \& McClelland, 2004). Where does the information driving this rapid acquisition come from? Undoubtedly, a sizeable component comes from direct experience observing and interacting with the world (Sloutsky \& Fisher, 2004; Stahl \& Feigenson, 2015). But another important source of information comes from the language people use to talk about the world (Landauer \& Dumais, 1997; Rhodes, Leslie, \& Tworek, 2012). How similar is the information available from children's direct experience to the information available in the language children hear?

Two lines of work suggest that they may be surprisingly similar. One compelling area of work is the comparison of semantic structures learned by congenitally blind children to those of their sighted peers. In several domains that would at first blush rely heavily on visual information, such as verbs of visual perception (e.g., look, see), blind children and adults make semantic similarity judgments that mirror their sighted peers (Bedny, Koster-Hale, Elli, Yazzolino, \& Saxe, 2019; Landau, Gleitman, \& Landau, 2009). A second line of evidence supporting the similarity of information in perception and language is the broad success of statistical models trained on language alone in approximating human judgments across a variety of domains (Landauer \& Dumais, 1997; Mikolov,
Sutskever, Chen, Corrado, \& Dean, 2013). Even more compellingly, models trained on both language usage and perceptual features for some words can infer the perceptual features of linguistically related words entirely from the covariation of language and perception (Johns \& Jones, 2012).

Still, there is reason to believe that some semantic features may be harder to learn from language than these data suggest. This is because we rarely use language merely to provide running commentary on the world around us; instead, we use language to talk about things that diverge from our expectations or those of our conversational partner (Grice, 1975). People tend to avoid being over- or under-informative when they speak. In particular, when referring to objects, people are informative with respect to both the referential context and the typical features of the referent (Rubio-Fernández, 2016; Westerbeek, Koolen, \& Maes, 2015). People tend to refer to an object that is typical of its category with a bare noun (e.g., calling an orange carrot "a carrot"), but often specify when an object has an atypical feature (e.g, "a purple carrot"). Given these communicative pressures, naturalistic language statistics may provide surprisingly little evidence about what is typical (Willits, Sussman, \& Amato, 2008).

If parents speak to children in this minimally informative way, children may be faced with input that emphasizes atypicality in relation to world knowledge they do not yet have. For things like carrots-which children learn about both from perception and from language-this issue may be resolved by integrating both sources of information. Likely almost all of the carrots children see are orange, and hearing an atypical exemplar noted as a "purple carrot" may make little difference in their inferences about the category of carrots more broadly. But for things to which they lack perceptual accesssuch as rare objects, unfamiliar social groups, or inaccessible features like the roundness of the Earth-much of the information must come from language (Harris \& Koenig, 2006). If language predominantly notes atypical features rather than typical ones, children may overrepresent atypical features as they learn the way things in the world tend to be.

On the other hand, parents may speak to children far differently from the way they speak to other adults. Parents' speech may reflect typical features of the world more veridically, or even emphasize typical features in order to teach children about the world. Parents alter their speech to children along a number of structural dimensions, using simpler 


\begin{tabular}{llrrrrr}
\hline utterance & pair & rating 1 & rating 2 & rating 3 & rating 4 & mean typicality \\
\hline especially with wooden shoes. & wooden-shoe & 2 & 2 & 3 & 2 & 2.25 \\
you like red onions? & red-onion & 3 & 5 & 3 & 4 & 3.75 \\
the garbage is dirty. & dirty-garbage & 7 & 7 & 5 & 7 & 6.50 \\
\hline
\end{tabular}

Table 1: Sample typicality ratings from 4 human coders for three adjective-noun pairs drawn from the corpus.

syntax and more reduplications (Snow, 1972). Their use of description may reflect similar alignment to children's growing knowledge.

We examine the typicality of adjectives in a large, diverse corpus of parent-child interactions recorded in children's homes to ask whether parents talking to their children tend to use adjectives predominantly to mark atypical features. We find that they do: Parents and children overwhelmingly choose to mention atypical rather than typical features. We also find that parents use adjectives differently over the course of children's development, noting typical features more often to younger children. We then ask whether the co-occurrence structure of language nonetheless captures typicality information by training vector space models on child-directed speech. We find that relatively little typical feature information is represented in these semantic spaces.

\section{Adjective typicality}

In order to determine whether parents use adjectives mostly to mark atypical features of categories, we analyzed caregiver speech from a large corpus of parent-child interactions. We extracted a subset of adjective-noun combinations that co-occurred, and asked a sample of Amazon Mechanical Turkers to judge how typical the property described by each adjective was for the noun it modified. We then examined both the broad features of this typicality distribution and the way it changes over development. Our theoretical hypotheses, statistical models, sample size, and exclusion criteria were pre-registered on the Open Science Framework (https://osf.io/ypdzv/).

\section{Corpus}

We used data from the Language Development Project, a large-scale, longitudinal corpus of parent-child interactions recorded in children's homes. Families were recruited to be representative of the Chicagoland area in both socioeconomic and racial composition (Goldin-Meadow et al., 2014). Recordings were taken in the home every 4 months from when the child was 14 months old until they were 58 months old, resulting in 12 timepoints. Each recording was of a 90-minute session in which parents and children were free to behave and interact as they liked.

Our sample consisted of 64 typically-developing children and their caregivers with data from at least 4 timepoints (mean $=11.3$ timepoints). Together, this resulted in a total of 641,402 distinct parent utterances.

\section{Stimulus Selection}

From these utterances, we extracted all of the nouns (using human-coded part of speech tags) resulting in a set of 8,150 total nouns. Because of our interest in change over development, we considered only nouns that appeared at least once every 3 sessions (i.e. per developmental year). This yielded a set of some 1,829 potential target nouns used over 198,014 distinct utterances.

We selected from the corpus all 35,761 distinct utterances containing any of these nouns and any word tagged as an adjective. We considered for analysis all adjective-noun pairs that occurred in any utterance (e.g., utterances with one noun and three adjectives were coded as three pairs) for a total of 18,050 distinct pairs. This set contained a number of highfrequency idiomatic pairs whose typicality was difficult to classify (e.g., "good"-"job"; "little"-"bit"). To resolve this issue, we used human judgments of words' concreteness to identify and exclude candidate idioms (Brysbaert, Warriner, \& Kuperman, 2014). We retained for analysis only pairs in which both the adjective and noun were in the top $25 \%$ of the concreteness ratings (e.g., "dirty" - "dish"; "green" - "fish") restricting our set to 2,477. Finally, human coders in the lab judged whether each pair was "incoherent or unrelated" and we excluded a final 576 pairs from the sample (e.g., incoherent pairs such as "flat" - "honey").

Thus, our final sample included 1,901 unique adjectivenoun pairs drawn from 3,749 distinct utterances. The pairs were combinations of 637 distinct concrete nouns and 111 distinct concrete adjectives. We compiled these pairs and collected human judgments on Amazon Mechanical Turk for each pair, as described below. Table 1 contains example utterances from the final set and typicality judgments from our human raters.

\section{Participants}

Each participant rated 20 adjective-noun pairs, and each pair was rated by four participants; we used Dallinger, a tool for automating complex recruitment on Amazon Mechanical Turk, to balance recruitment. Overall, we recruited 444 participants to rate our final sample of adjective-noun pairs. After exclusions using an attention check that asked participants to simply choose a specific number on the scale, we retained 8,580 judgments, with each adjective-noun pair retaining at least two judgments.

\section{Design and Procedure}

To evaluate the typicality of the adjective-noun pairs that appeared in parents' speech, we asked participants on Amazon 
Mechanical Turk to rate each pair. Participants were presented with a question of the form "How common is it for a cow to be a brown cow?" and asked to provide a rating on a seven-point scale: (1) never, (2) rarely, (3) sometimes, (4) about half the time, (5) often, (6) almost always, (7) always.

\section{Results}

The human typicality ratings were combined with usage data from our corpus analysis to let us determine the extent to which parents use language to describe typical and atypical features. In our analyses, we token-weighted these judgments, giving higher weight to pairs that occurred more frequently in children's inputs. However, results are qualitatively identical and all significant effects remain significant without these re-weightings.

If caregivers speak informatively to convey what is atypical or surprising in relation to their own sophisticated world knowledge, we should see that caregiver description is dominated by modifiers that are sometimes or rarely true of the noun they modify. If instead child-directed speech privileges redundant information, perhaps to align to young children's limited world knowledge, caregiver description should yield a distinct distribution dominated by highly typical modifiers. As predicted in our pre-registration, we find that parents' description predominantly focuses on features that are atypical (Figure 1).

To confirm this effect statistically, we centered the ratings (i.e. "about half" was coded as 0 ), and then predicted the rating on each trial with a mixed effect model with only an intercept and a random effect of noun (typicality $\sim 1+$ $(1 \mid$ noun $))$. The intercept was reliably negative, indicating that adjectives tend to refer to atypical features of objects $(\beta=-0.77, t=-19.72, p<.001)$. We then re-estimated these models separately for each age in the corpus, and found a reliably negative intercept for every age group (smallest effect $\beta_{14}=-0.5, t=-4.45, p=<.001$ ). These data suggest that even when talking with very young children, caregiver speech is structured according to adult communicative pressures observed in the lab.

For comparison, we performed the same analyses but with typicality judgments weighted not by the frequency of each adjective-noun pair's occurrence in the Language Development Project, but instead by their frequency of occurrence in the Corpus of Contemporary American English (COCA; Davies, 2008). While this estimate of adult usage is imperfect - the adjective-nouns pairs produced by parents in our corpus may not be a representative sample of adjectives and nouns spoken by the adults in COCA-it provides a first approximation to adult usage. When we fit the same mixed-effects model to the data, we found that the intercept was reliably negative, indicating that adult-to-adult speech is likely also biased toward description of atypical features $(\beta=$ $-0.3, t=-19.72, p<.001)$.

Returning to caregiver speech, while descriptions at every age tended to point out atypical features (as in adult-to-adult speech), this effect changed in strength over development. As

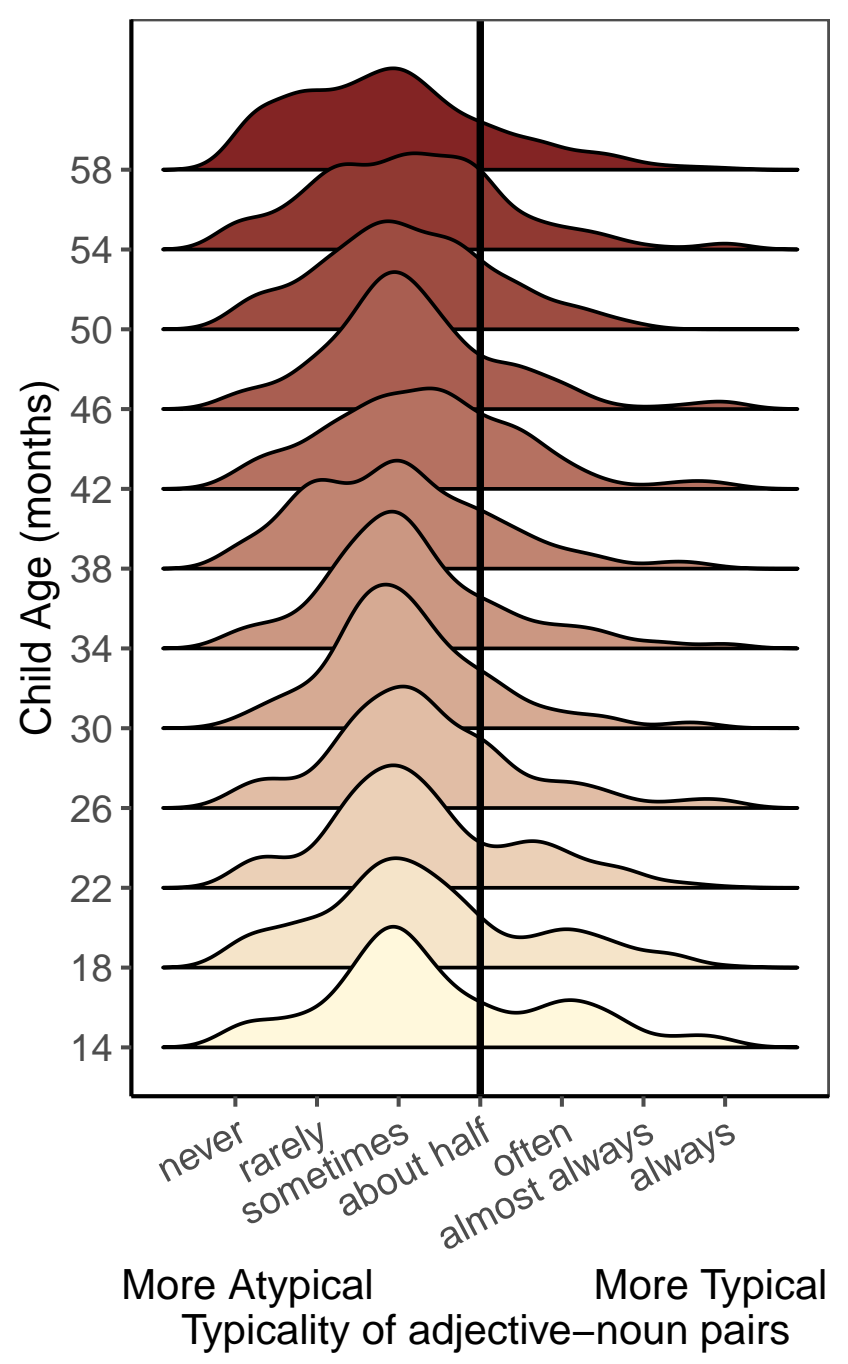

Figure 1: Density plots showing usage at each timepoint based on the typicality of the adjective-noun pair.

predicted, an age effect added to the previous model was reliably negative, indicating that parents of older children are relatively more likely to focus on atypical features $(\beta=-0.11$, $t=-3.47, p=.001)$. In line with the idea that caregivers adapt their speech to their children's knowledge, it seems that caregivers are more likely to provide description of typical features for their young children, compared with older children. As a second test of this idea, we defined adjectives as highly typical if Turkers judged them to be 'often', 'almost always', or 'always' true. We predicted whether each judgment was highly typical from a mixed-effects logistic regression with a fixed effect of age (log-scaled) and a random effect of noun. Age was a highly reliable predictor $(\beta=-0.94, t=-5.01, p=$ $<.001)$. While children at all ages hear more talk about what is atypically true (Figure 1), younger children hear relatively more talk about what is typically true than older children do (Figure2). 


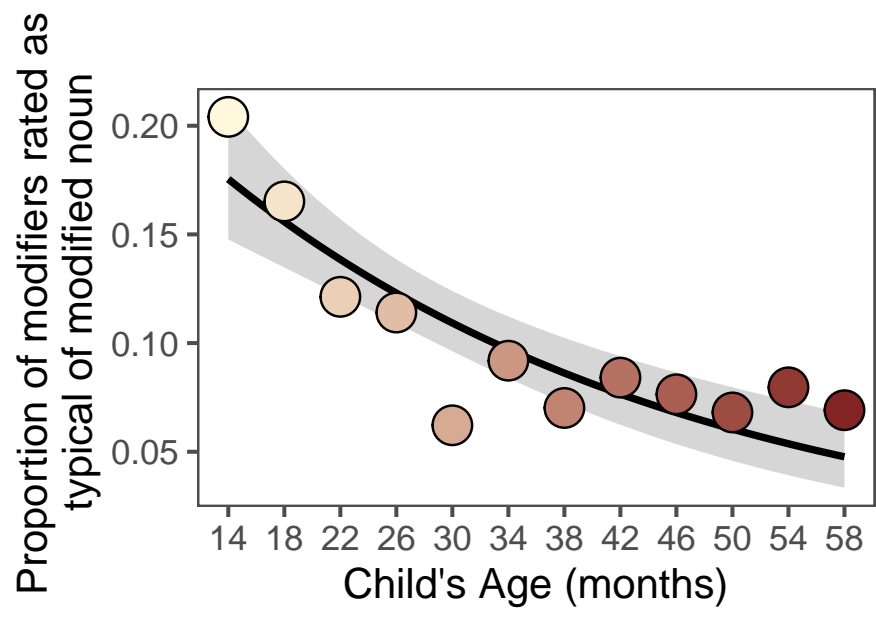

Figure 2: Proportion of caregiver description that is about typically-true features, as a function of age.

Child Speech. Given the striking consistency in adult-toadult speech and caregiver speech across ages, we next briefly consider what kind of information is contained in children's speech. By analyzing children's own utterances, we can determine when children come to use description in a way that looks like adult speech. Are children mirroring adult-like uses of description even from a young age, or are they choosing to describe more typical features of the world?

The Language Development Corpus contains 368,348 child utterances. Using the set of adjective-noun pairs for which we have judgments from our analysis of caregiver speech, we repeat our analysis on usage data for a set of 460 distinct adjective-noun pairs which also appeared in children's productions. While preliminary, a mixed effects model predicting typicality had a highly-reliable negative intercept ( $\beta=-0.72, t=-10.14, p=<.001)$, but adding an age term did not improve model fit. Thus, children's speech is also biased towards atypical descriptions, and this bias does not change reliably over the first 5 years.

\section{Discussion}

In sum, we find robust evidence that language is used to discuss atypical, rather than typical, features of the world. Description in caregiver speech seems to largely mirror the usage patterns that we observed in adult-to-adult speech, suggesting that these patterns arise from general communicative pressures. Indeed, even children's own productions show a similar usage pattern, with more description of atypical features of the world even at the youngest ages.

It should be noted that children's utterances come from naturalistic conversations with caregivers, and their use of atypical description may be prompted by parent-led discourse. That is, if a caregiver chooses to describe the purpleness of a cat in book, the child may well respond by asking about that same feature. Further, atypical descriptors may actually be more likely to elicit imitation from child speakers, compared with typical descriptors (Bannard, Rosner, \&
Matthews, 2017). Future analyses would need to better disentangle the extent to which children's productions are imitative of caregivers.

Interestingly, the descriptions children hear change over development, becoming increasingly focused on atypical features. The higher prevalence of typical descriptors in early development may help young learners learn what is typical; however, even at the earliest point we measured, the bulk of language input describes atypical features.

This usage pattern aligns with the idea that language is used informatively in relation to background knowledge about the world. It may pose a problem, however, for young language learners with still-developing world knowledge. If language does not transparently convey the typical features of objects, and instead (perhaps misleadingly) notes the atypical ones, how might children come to learn what objects are typically like? One possibility is that information about typical features is captured in regularities across many utterances. If this is true, language may still be an important source of information about typicality as children may be able to extract more accurate typicality information by tracking secondorder co-occurrence.

\section{Extracting Typicality from Language Structure}

Much information can be gleaned from language that does not seem available at first glance. From language alone, simple distributional learning models can recover enough information to perform comparably to non-native college applicants on the Test of English as a Foreign Language (Landauer \& Dumais, 1997). Recently, Lewis, Zettersten, \& Lupyan (2019) demonstrated that even nuanced feature information may be learnable through distributional semantics alone, without any complex inferential machinery. We take a similar approach to ask whether a distributional semantics model trained on the language children hear can capture typical feature information.

\section{Method}

To test this possibility, we trained word2vec-a distributional semantics model-on the same corpus of child-directed speech used in our first set of analyses. Word2vec is a neural network model that learns to predict words from the contexts in which they appear. This leads word2vec to learn representations in which words that appear in similar contexts become similar to each-other (Firth, 1957).

We used the continuous-bag-of-words (CBOW) implementation of word2 vec in the gensim package (Řehưřek \& Sojka, 2010). We trained the model using a surrounding context of 5 words on either side of the target word and 100 dimensions (weights in the hidden layer) to represent each word. After training, we extracted the hidden layer representation of each word in the model's vocabulary-these are the vectors used to represent these words.

If the model captures information about the typical features of objects, we should see that the model's noun-adjective 
word pair similarities are correlated with the typicality ratings we elicited from human raters. For a second comparison, we also used an off-the-shelf implementation of word2vec trained on Wikipedia (Mikolov, Grave, Bojanowski, Puhrsch, \& Joulin, 2018). While the Language Development Project corpus likely underestimates the amount of structure in children's linguistic input, Wikipedia likely overestimates it.

\section{Results}

We find that similarities in the model trained on the Language Development Project corpus have near zero correlation with human adjective-noun typicality ratings $(r=0.03, p=.208)$. However, our model does capture other meaningful information about the structure of language, such as similarity. Comparing with pre-existing large-scale human similarity judgements for word pairs, our model shows significant correlations (correlation with wordsim353 similarities of noun pairs, 0.28 ; correlation with simlex similarities of noun, adjective, and verb pairs, 0.16). This suggests that statistical patterns in child-directed speech are likely insufficient to encode information about the typical features of objects, despite encoding at least some information about word meaning more broadly.

However, the corpus on which we trained this model was small; perhaps our model did not get enough language to draw out the patterns that would reflect the typical features of objects. To test this possibility, we asked whether word vectors trained on a much larger corpus-English Wikipediastrongly correlate with typicality ratings. This model's similarities were significantly correlated with human judgments, although the strength of the correlation was still fairly weak $(r=0.25, p<.001)$. Interestingly, similarities from the two models correlated more highly to each other than either model correlated with human judgments $(r=0.29, p<.001)$. This suggests that these models are picking up on some systematic associations between nouns and adjectives, but not the typical features of things.

One possible confound in these analyses is that the similarity judgments produced by our models reflect many dimensions of similarity, but our human judgments reflect only typicality. To accommodate this, we performed a second analysis in which we considered only the subset of 73 nouns that had both a typical (rated as at least "often") and an atypical (rated as at most "sometimes") adjective. We then asked whether the models rated the typical adjective as more similar to the noun it modified than the atypical adjective. The LDP model correctly classified 38 out of $73(0.52)$, which was not better than chance $(p=.815)$. The Wikipedia model correctly classified 56 out of 73 (0.77), which was better than chance according to a binomial test, but still fairly poor performance $(p=<.001)$. Fig 3 shows the ratings from Turkers and the two models for the 73 nouns. Table 2 gives the six cases in which word2vec similarities are worst at predicting human typicality judgments, judging the low-typicality adjective to be more similar to the noun than the high-typicality adjective.

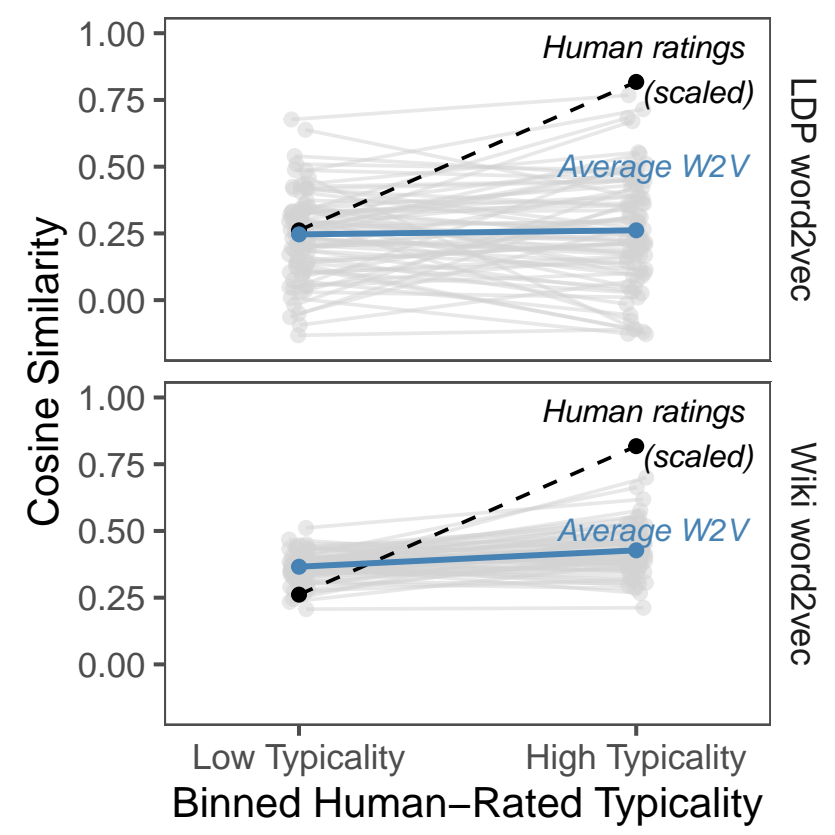

Figure 3: Plots of word2vec noun-adjective similarities for nouns for which there was at least one atypical adjective (rated at most "sometimes"), and at least one typical adjective (rated at least"often").

\section{General Discussion}

Language provides children a rich source of information about the world. However, this information is not always transparently available: because language is used to comment on the atypical, it does not perfectly mirror the world. Among adult conversational partners whose world knowledge is well-aligned, this allows people to converse informatively and avoid redundancy. But between a child and caregiver whose world knowledge is asymmetric, this pressure competes with other demands: what is minimally informative to an adult may be misleading to a child. Our results show that this pressure structures language to create a peculiar learning environment, one in which caregivers predominantly point out the atypical features of things.

\begin{tabular}{lll}
\hline noun & typical adjective & atypical adjective \\
\hline puzzle & flat & giant \\
apple & red & brown \\
bird & outside & purple \\
elephant & fat & pink \\
whale & wet & red \\
frog & green & purple \\
\hline
\end{tabular}

Table 2: The top six cases in which Wikipedia-trained word2vec similarities were worst at predicting human typicality judgments. In each case, word2vec judged the lowtypicality adjective to be more similar to the noun than the high-typicality adjective. 
How, then, do children learn about the typical features of things? While younger children may gain an important foothold from hearing more description of typical features, they still face language dominated by atypical description. When we looked at more nuanced ways of extracting information from language (which may or may not be available to the developing learner), we found that models of distributional semantics capture little typical feature information.

Of course, perceptual information from the world may simplify this problem. In many cases, perceptual information may swamp information from language; children likely see enough orange carrots in the world to outweigh hearing "purple carrot." It remains unclear, however, how children learn about categories for which they have scarcer evidence. Indeed, language information likely swamps perceptual information for many other categories, such as abstract concepts or those that cannot be learned about by direct experience. If such concepts pattern similarly to the concrete objects analyzed here, children are in a particularly difficult bind.

It is also possible that other cues from language and interaction provide young learners with clues to what is typical or atypical, and these cues are uncaptured by our measure of usage statistics. Caregivers may highlight when a feature is typical by using certain syntactic constructions, such as generics (e.g., "tomatoes are red"). Caregivers may also mark the atypicality of a feature, for example demonstrating surprise. Such cues from language and the interaction may provide key information in some cases; however, given the sheer frequency of atypical descriptors, it seems unlikely that they are consistently well-marked.

Another possibility is that children expect language to be used informatively at a young age. Under this hypothesis, their language environment is not misleading at all, even without additional cues from caregivers. Children as young as two years old tend to use words to comment on what is new rather than what is known or assumed (Baker \& Greenfield, 1988). Children may therefore expect adjectives to comment on surprising features of objects. If young children expect adjectives to mark atypical features (Horowitz \& Frank, 2016), they can use description and the lack thereof to learn more about the world. Indeed, this idea is consistent with our finding that even young children largely choose to describe atypical features. Though this effect can be explained by simpler means such as mimicry, it suggests that caregivers and children may be usefully aligned in the aspects of the world they choose to talk about.

Whether adult-directed, child-directed, or a child's own speech, language is used with remarkable consistency: people talk about the atypical. Though parents might reasonably be broadly over-informative in order to teach their children about the world, this is not the case. This presents a potential puzzle for young learners who have limited world knowledge and limited pragmatic inferential abilities. Perceptual information and nascent pragmatic abilities may help fill in the gaps, but much remains to be explored to link these ex- planations to actual learning. Communication pressures are pervasive forces structuring the language children hear, and future work must disentangle whether children capitalize on them or are misled by them in learning about the world.

Stimuli, data, and analysis code available at https://osf.io/ypdzv/

\section{Acknowledgements}

This research was funded by a James S. McDonnell Foundation Scholar Award to DY.

\section{References}

Baillargeon, R. (1994). How do infants learn about the physical world? Current Directions in Psychological Science, 3(5), 133-140.

Baker, N. D., \& Greenfield, P. M. (1988). The development of new and old information in young children's early language. Language Sciences, 10(1), 3-34.

Bannard, C., Rosner, M., \& Matthews, D. (2017). What's worth talking about? Information theory reveals how children balance informativeness and ease of production. Psychological Science, 28(7), 954-966.

Bedny, M., Koster-Hale, J., Elli, G., Yazzolino, L., \& Saxe, R. (2019). There's more to "sparkle" than meets the eye: Knowledge of vision and light verbs among congenitally blind and sighted individuals. Cognition, 189, 105-115.

Brysbaert, M., Warriner, A. B., \& Kuperman, V. (2014). Concreteness ratings for 40 thousand generally known english word lemmas. Behavior Research Methods, 46(3), 904-911.

Davies, M. (2008). The corpus of contemporary american english (coca): 520 million words, 1990-present.

Firth, J. R. (1957). A synopsis of linguistic theory, 19301955. Studies in Linguistic Analysis.

Goldin-Meadow, S., Levine, S. C., Hedges, L. V., Huttenlocher, J., Raudenbush, S. W., \& Small, S. L. (2014). New evidence about language and cognitive development based on a longitudinal study: Hypotheses for intervention. American Psychologist, 69(6), 588.

Grice, H. P. (1975). Logic and conversation. In Speech acts (pp. 41-58). Brill.

Harris, P. L., \& Koenig, M. A. (2006). Trust in testimony: How children learn about science and religion. Child Development, 77(3), 505-524.

Horowitz, A. C., \& Frank, M. C. (2016). Children's pragmatic inferences as a route for learning about the world. Child Development, 87(3), 807-819.

Johns, B. T., \& Jones, M. N. (2012). Perceptual inference through global lexical similarity. Topics in Cognitive Science, 4(1), 103-120.

Landau, B., Gleitman, L. R., \& Landau, B. (2009). Language and experience: Evidence from the blind child (Vol. 8). Harvard University Press. 
Landauer, T. K., \& Dumais, S. T. (1997). A solution to plato's problem: The latent semantic analysis theory of acquisition, induction, and representation of knowledge. Psychological Review, 104(2), 211.

Legare, C. H., \& Harris, P. L. (2016). The ontogeny of cultural learning. Child Development, 87(3), 633-642.

Lewis, M., Zettersten, M., \& Lupyan, G. (2019). Distributional semantics as a source of visual knowledge. Proceedings of the National Academy of Sciences, 116(39), 1923719238.

Mikolov, T., Grave, E., Bojanowski, P., Puhrsch, C., \& Joulin, A. (2018). Advances in pre-training distributed word representations. In Proceedings of the international conference on language resources and evaluation (lrec 2018).

Mikolov, T., Sutskever, I., Chen, K., Corrado, G. S., \& Dean, J. (2013). Distributed representations of words and phrases and their compositionality. In Advances in neural information processing systems (pp. 3111-3119).

Rhodes, M., Leslie, S.-J., \& Tworek, C. M. (2012). Cultural transmission of social essentialism. Proceedings of the $\mathrm{Na}$ tional Academy of Sciences, 109(34), 13526-13531.

Rogers, T. T., \& McClelland, J. L. (2004). Semantic cognition: A parallel distributed processing approach. MIT press.

Rubio-Fernández, P. (2016). How Redundant Are Redundant Color Adjectives? An Efficiency-Based Analysis of Color Overspecification. Frontiers in Psychology, 7.

Řehůřek, R., \& Sojka, P. (2010). Software Framework for Topic Modelling with Large Corpora. In Proceedings of the LREC 2010 Workshop on New Challenges for NLP Frameworks (pp. 45-50). Valletta, Malta: ELRA.

Sloutsky, V. M., \& Fisher, A. V. (2004). Induction and categorization in young children: A similarity-based model. Journal of Experimental Psychology: General, 133(2), 166.

Snow, C. E. (1972). Mothers' speech to children learning language. Child Development, 549-565.

Stahl, A. E., \& Feigenson, L. (2015). Observing the unexpected enhances infants' learning and exploration. Science, 348(6230), 91-94.

Westerbeek, H., Koolen, R., \& Maes, A. (2015). Stored object knowledge and the production of referring expressions: The case of color typicality. Frontiers in Psychology, 6.

Willits, J. A., Sussman, R. S., \& Amato, M. S. (2008). Event knowledge vs. Verb knowledge. In Proceedings of the 30 th annual conference of the cognitive science society (pp. 2227-2232). 